\title{
Formation of crystalline silicate around oxygen-rich AGB stars
}

\author{
Takashi Kozasa and Hisato Sogawa \\ Department of Earth and Planetary Sciences, Kobe University, \\ Kobe 657-8501, Japan
}

\begin{abstract}
Crystallization of silicate has been investigated within the framework of dust formation in steady state gas outflows around oxygenrich AGB stars, where silicates are locked not only into homogeneous silicate grains but also into the mantles of heterogeneous grains. Based on the thermal history of dust grains after their formation, the crystallization calculation results in no crystalline silicate for the mass loss rate $\dot{M} \leq 2 \times$ $10^{-5} M_{\odot} \mathrm{yr}^{-1}$. Only silicate in the mantles of heterogeneous grains can be crystallized for $\dot{M} \geq 3 \times 10^{-5} M_{\odot} \mathrm{yr}^{-1}$, while homogeneous silicate grains remain amorphous. The mass fraction of crystalline silicate increases with increasing $\dot{M}$. The radiation transfer calculations confirm the appearance of an emission feature around $33.5 \mu \mathrm{m}$, taking olivine as a representative of crystalline silicates. On the other hand, the $10 \mu \mathrm{m}$ feature appears in absorption, being dominated by homogeneous silicate grains. These trends are consistent with the observations. Thus the crystalline silicate is a diagnostics of high mass loss rate at the late stage of AGB stellar evolution, reflecting the formation process of dust grains.
\end{abstract}

\section{Introduction}

The ISO-SWS observations revealed the presence of crystalline silicate in circumstellar envelopes of oxygen-rich evolved stars from emission features between 25 and $45 \mu \mathrm{m}$ (Waters et al. 1996). Cami et al. (1998) have shown that oxygen-rich AGB stars with high mass loss rate exhibit the prominent emission features of crystalline silicates with absorption features around 10 and $18 \mu \mathrm{m}$; otherwise no crystalline silicate feature is seen in AGB stars with low mass loss rate. The $10 \mu \mathrm{m}$ absorption features analyzed so far show no obvious structure due to crystalline silicates, being dominated by amorphous silicates (Tielens et al. 1998). The peak positions of the emission features suggest that crystalline silicates are $\mathrm{Mg}$-rich olivine and pyroxene, although amorphous silicates have been considered to be Fe-rich (Tielens et al. 1998, and references therein).

The observed characteristics of silicate features seem to be closely related to the formation process of dust grains through the mass loss rate of AGB stars. Although Tielens et al. (1998) addressed the formation scenario of crystalline silicate, the details are still uncertain. In this paper we explore the origin and formation process of crystalline silicate within the framework of dust formation around oxygen-rich AGB stars. Summarizing the model of dust formation and 

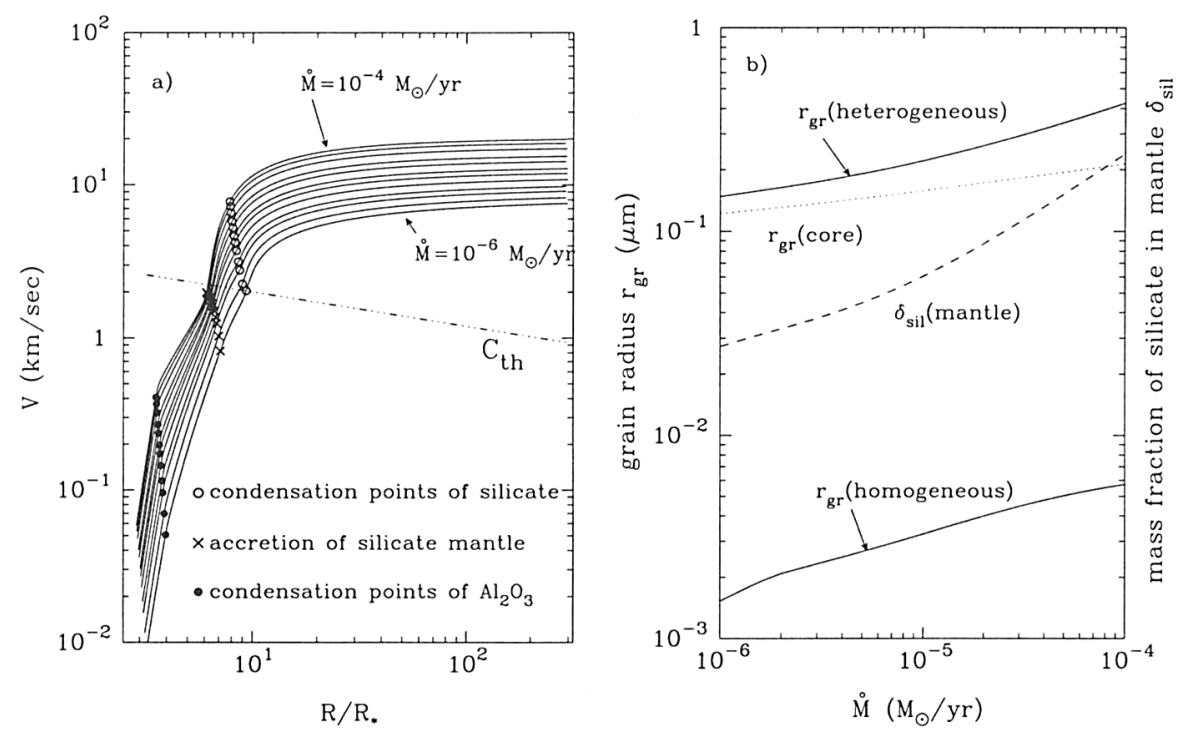

Figure 1. a) The gas outflow solutions and the locations of dust condensation for $\dot{M}=10^{-6}$ to $10^{-4} M_{\odot} /$ yr. b) The final average radii of dust grains (solid and dotted lines) and the mass fraction of silicate locked into the mantle of heterogeneous grains (dashed line) versus $\dot{M}$.

introducing a kinetic theory of crystallization, we shall show that crystalline silicate is a natural product when the mass loss rate is large enough.

\section{Dust formation and the thermal history of dust grains}

We have developed a model of dust formation in steady state dust driven winds around oxygen-rich AGB stars (see Kozasa \& Sogawa 1997, 1998). The model considers the formation of not only homogeneous corundum $\left(\mathrm{Al}_{2} \mathrm{O}_{3}\right)$ and silicate grains but also heterogeneous grains consisting of an $\mathrm{Al}_{2} \mathrm{O}_{3}$ core and a silicate mantle simultaneously. The radial dependence of the gas temperature is assumed to be given by $T(R)=T_{*}\left(R / R_{*}\right)^{-\beta}$ where $R_{*}$ is the stellar radius and $T_{*}$ the effective temperature. In the present calculations, the stellar parameters are $M_{*}=1.0 M_{\odot}, L_{*}=2 \times 10^{4} L_{\odot}$ and $T_{*}=2500 \mathrm{~K}$. The mass loss rate $\dot{M}$ ranges from $10^{-6}$ to $10^{-4} M_{\odot} \mathrm{yr}^{-1}$, and $\beta=0.45$.

The locations of condensation of dust grains and the resulting gas outflows are depicted in Fig. 1a, where the condensation point means the location at which the nucleation rate reaches its maximum. The accretion of silicate on pre-condensed $\mathrm{Al}_{2} \mathrm{O}_{3}$ grains starting slightly inside the sonic point drives the gas outflow into the supersonic region. Beyond the sonic point, homogeneous silicate grains condense. The final average radii of heterogeneous grains range from 0.15 to $0.4 \mu \mathrm{m}$ (see Fig. 1b). Homogeneous silicate grains are very small: $r_{\mathrm{gr}}=15$ to $60 \AA$. The mass fraction of silicate locked into the mantles of heterogeneous grains $\delta_{\text {sil }}$ increases with increasing $\dot{M}$, and reaches 0.1 for $\dot{M}=3 \times 10^{-5} M_{\odot} \mathrm{yr}^{-1}$. 

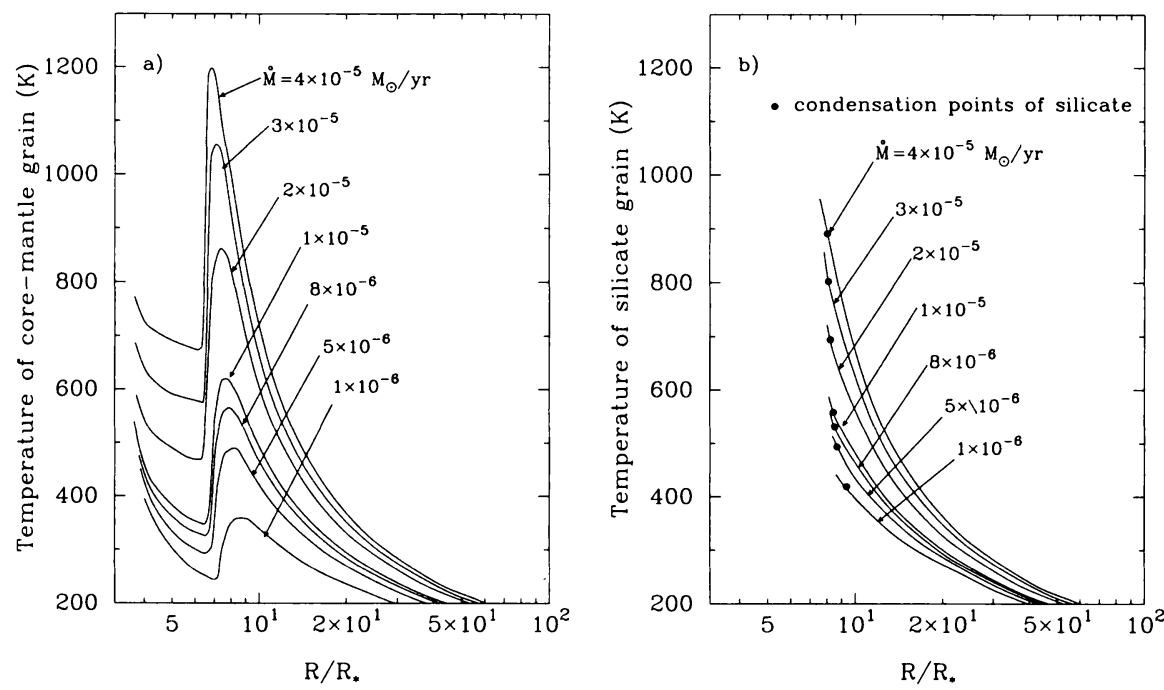

Figure 2. Temperatures of dust grains after their formation for the given mass loss rates; a) for $\mathrm{Al}_{2} \mathrm{O}_{3}$ and heterogeneous grains, and b) for homogeneous silicate grains

Fig. 2 shows the temperatures of dust grains after their formation for $\dot{M} \leq 4 \times 10^{-5} M_{\odot} \mathrm{yr}^{-1}$. They are calculated by a radiation transfer code using a Monte-Carlo method (Fig. 2a: $\mathrm{Al}_{2} \mathrm{O}_{3}$ and heterogeneous grains, Fig. $2 \mathrm{~b}$ : homogeneous silicate grains). The temperatures of $\mathrm{Al}_{2} \mathrm{O}_{3}$ grains are very low, reflecting the transparency of $\mathrm{Al}_{2} \mathrm{O}_{3}$ in the visible and near infrared region. As an $\mathrm{Al}_{2} \mathrm{O}_{3}$ grain is coated with an absorptive silicate mantle, the temperature of heterogeneous grain increases steeply, reaches a maximum and then decreases with increasing $R$. The maximum temperature is higher than 1000 $\mathrm{K}$ for $\dot{M} \geq 3 \times 10^{-5} M_{\odot} \mathrm{yr}^{-1}$. On the other hand, with the temperature $\leq 900$ $\mathrm{K}$ at the condensation point, the temperatures of homogeneous silicate grains monotonically decrease.

At the time of accretion of silicate, the temperatures of $\mathrm{Al}_{2} \mathrm{O}_{3}$ cores are much lower than the glass temperature of silicate ( $\sim 1050 \mathrm{~K}$, see Tielens et al. 1998). Also the condensation temperature of homogeneous silicate is lower than $1000 \mathrm{~K}$ (Kozasa \& Sogawa 1997, 1998). Thus newly formed silicates are to be amorphous. The thermal history of dust grains after their formation is crucial in determining the crystallinity of silicate as discussed in the following sections.

\section{A kinetic theory of crystallization of amorphous materials}

Crystallization of amorphous solid proceeds through the spontaneous nucleation of crystalline nuclei and the following growth during heating and/or cooling processes. The diffusion of molecules controls the kinetics. The steady state nucleation rate $J$ and the growth rate $w$ for formation of spherical crystal are given by 


$$
\begin{gathered}
J(t)=\frac{2 \pi}{\Omega}\left(\frac{\sigma}{k T}\right)^{1 / 2} \frac{D_{0}}{\Omega^{2 / 3}} \exp \left[-\frac{1}{k T}\left(E_{\mathrm{a}}+\frac{16 \pi}{3} \frac{\sigma^{3}}{\Delta G_{\mathrm{v}}^{2}}\right)\right] \\
\omega(t)=\frac{D_{0}}{\Omega^{1 / 3}} \exp \left(-\frac{E_{\mathrm{a}}}{k T}\right)\left[1-\exp \left(-\frac{\Delta G_{\mathrm{v}}}{k T}\right)\right]
\end{gathered}
$$

where $\Omega$ is the molecular volume, $\sigma$ the interfacial energy and $\Delta G_{\mathbf{v}}$ the Gibbs free energy change per molecule between amorphous and crystalline phases (see Kouchi et al. 1994 for the details). The diffusion coefficient is written by $D=$ $D_{0} \exp \left(-E_{\mathrm{a}} / k T\right)$ with $E_{\mathrm{a}}$ the activation energy. Then the temporal evolution of crystallized volume fraction $\theta(t)$ during heating and/or cooling processes is expressed by the standard and widely used "Avrami equation" (Avrami 1939)

$$
1-\theta(t)=\exp \left[-\frac{4 \pi}{3} \int_{0}^{t} J\left(t^{\prime}\right)\left(\int_{t^{\prime}}^{t} \omega\left(t^{\prime \prime}\right) d t^{\prime \prime}\right)^{3} d t^{\prime}\right]
$$

In principle, applying the above equation for thermal history of dust grains after their formation, we can calculate the crystallized volume fraction of dust grains.

At present time the basic data such as $D_{0}, E_{a}$ and $\Delta G_{\mathbf{v}}$ are not available for crystallization of silicates. However, it is possible to estimate the necessary data by referring to the annealing experiments of "magnesium silicate smokes" by Hallenbeck et al. (1998). Monitoring the infrared spectrum during isothermal annealing, they obtained the Arrhenius plot for the stall time $t_{\text {stall }}$ at which the change of the spectral features stalls for a while (see Figs. 1 and 8 in their paper). The stall spectrum allows us to estimate $\theta \sim 0.2$ at the stall time by comparison with the calculated spectrum for mixtures of amorphous and crystalline silicates. For isothermal annealing, equation (3) is reduced to $1-\theta=\exp \left[-\left(t / \tau_{\mathrm{cr}}\right)^{4}\right]$ with the characteristic time scale of crystallization $\tau_{\text {cr }}$ defined by

$$
\tau_{\mathrm{cr}}=\left(\frac{3}{2 \pi^{2}}\right)^{1 / 4}\left(\frac{k T}{\sigma}\right)^{1 / 8} \frac{\Omega^{2 / 3}}{D_{0}} \exp \left[\frac{1}{k T}\left(E_{\mathrm{a}}+\frac{4 \pi}{3} \frac{\sigma^{3}}{\Delta G_{\mathrm{v}}^{2}}\right)\right]
$$

The estimated $\theta \sim 0.2$ at the stall time corresponds to $t_{\text {stall }}=0.7 \tau_{\mathrm{cr}}$. Fitting the Arrhenius plot with Eq. (4) leads to the basic data: $\ln D_{0}=1.47 \times 10^{2}$ (in cgs units $), E_{\mathrm{a}} / k=1.72 \times 10^{5} \mathrm{~K}, \sigma / \Omega^{2 / 3}=731 \mathrm{erg} \mathrm{cm}^{-2}, \Delta G_{\mathrm{v}} / k=1.85 \times 10^{4}(1-$ $T / 1830) \mathrm{K}$, where we used $\Omega=5.22 \times 10^{-23} \mathrm{~cm}^{3}$. We shall adopt these derived values for the following calculations, although the "magnesium silicate smokes" may not be necessarily representative of "astronomical silicate".

\section{Crystallization of silicate and the resulting spectral feature}

We apply the theory presented in $\S 3$ to investigate the crystallization of silicate. The characteristic time scale of crystallization $\tau_{\mathrm{cr}}$ of silicate is very sensitive to the grain temperature because of the large activation energy $E_{\mathbf{a}}$; for example, $\tau_{\mathrm{cr}}$ $=20 \mathrm{sec}$., 30 days and $\sim 10^{6}$ years for $T=1100,1000$, and $900 \mathrm{~K}$, respectively. If the maximum temperature experienced by dust grains is less than $900 \mathrm{~K}$, the crystallization of silicate is impossible during the lifetime of AGB stars. Thus, homogeneous silicate grains as well as silicate in the mantles of heterogeneous 

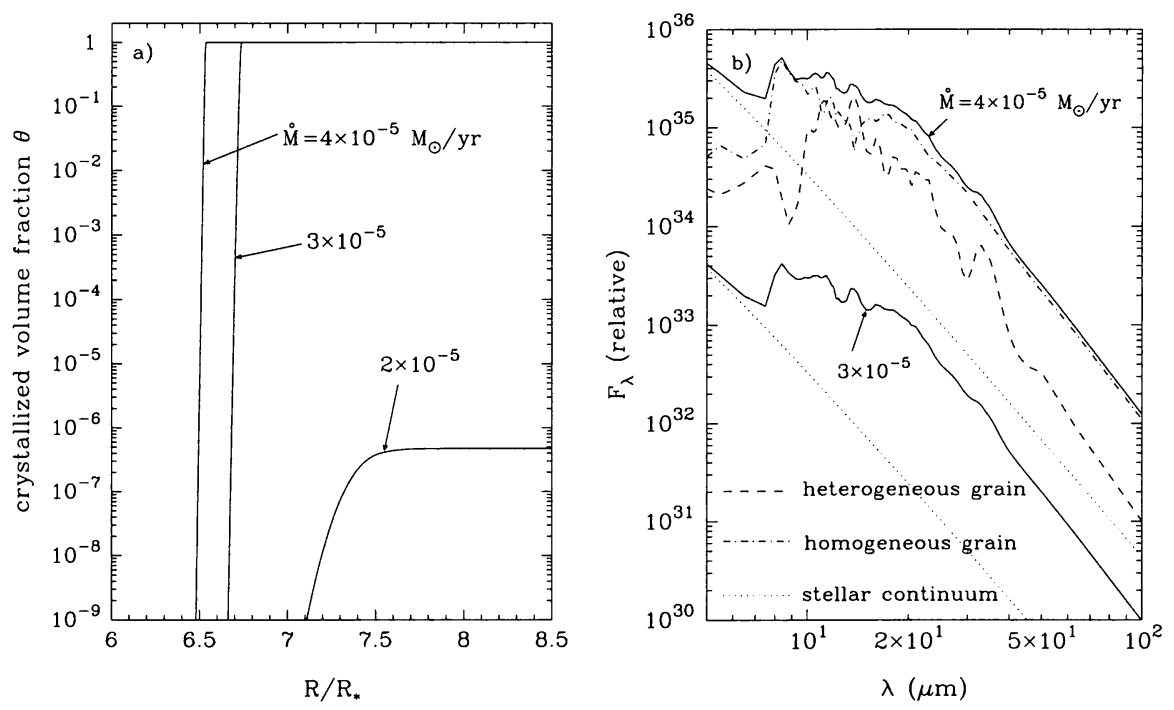

Figure $3 . \quad$ a) The spatial evolution of $\theta$ for silicate in the mantles of heterogeneous grains. b) The spectral energy distribution for the given mass loss rates. The contributions from the heterogeneous grains (dashed line) and the homogeneous silicate grains (dash-dotted line) to the total flux are indicated for $\dot{M}=4 \times 10^{-5} M_{\odot} / \mathrm{yr}$

grains can not be crystallized for $\dot{M} \leq 2 \times 10^{-5} M_{\odot} \mathrm{yr}^{-1}$ (see Fig. 2). Even if the temperature is higher than $900 \mathrm{~K}$, small homogeneous silicate grains can escape from crystallization due to the low probability of nucleation as discussed by Seki \& Hasegawa (1981).

Figure 3a shows the spatial evolution of the crystallized volume fraction of silicate in the mantles of heterogeneous grains calculated by Eq. (3) using the evolution of dust temperatures given in Fig. 2a. For $\dot{M}=2 \times 10^{-5} M_{\odot} \mathrm{yr}^{-1}$, crystallization is negligible $\left(\theta \leq 10^{-6}\right)$ as discussed above. On the other hand, silicate in the mantle is completely crystallized for $\dot{M} \geq 3 \times 10^{-5} M_{\odot} \mathrm{yr}^{-1}$ as the temperature steeply increases above $1000 \mathrm{~K}$. Thus only silicate in the mantles of heterogeneous grains can be crystallized for $\dot{M} \geq 3 \times 10^{-5} M_{\odot} \mathrm{yr}^{-1}$, while homogeneous silicate grains stay amorphous.

The spectral energy distributions calculated on the basis of the results of condensation and crystallization calculations are shown in Fig. 3b, where we take olivine as a representative of crystalline silicates and use the optical constants of $\left(\mathrm{Mg}_{0.9}, \mathrm{Fe}_{0.1}\right)_{2} \mathrm{SiO}_{4}$ (Koike 1998). First it should be noted that the continuum beyond $20 \mu \mathrm{m}$ is dominated by the thermal radiation from amorphous homogeneous-silicate grains as indicated for $\dot{M}=4 \times 10^{-5} M_{\odot} \mathrm{yr}^{-1}$. The distinguishable olivine feature appears around $33.5 \mu \mathrm{m}$ in emission over the continuum, while the $10 \mu \mathrm{m}$ feature appears in absorption and is dominated by homogeneous silicate grains. The emission feature of crystalline silicate becomes prominent with increasing $\dot{M}$ because $\delta_{\text {sil }}$ increases as presented in Fig. 2b, though the present calculation is restricted to $\dot{M} \leq 4 \times 10^{-5} M_{\odot} \mathrm{yr}^{-1}$. These 
trends of the appearance of silicate features are consistent with the observational results.

\section{Concluding remarks}

The origin and formation process of crystalline silicate were explored in the framework of dust formation in circumstellar envelopes of oxygen-rich AGB stars. The results of the calculations show that the carriers of crystalline silicate are heterogeneous grains consisting of an $\mathrm{Al}_{2} \mathrm{O}_{3}$ core and a silicate mantle. When the mass loss rate $\dot{M}$ is larger than $3 \times 10^{-5} M_{\odot} \mathrm{yr}^{-1}$, the crystallization occurs through the thermal processing due to heating caused by the accretion of silicate on $\mathrm{Al}_{2} \mathrm{O}_{3}$ grains starting slightly inside the sonic point. On the other hand, small homogeneous silicate grains condensing beyond the sonic point remain amorphous.

The behavior of calculated silicate features is consistent with that revealed by the observations. The derived lower limit of the mass fraction of crystalline silicate is 0.1 , which is larger than the value $\sim 0.05$ derived from the observations (Tielens et al. 1998). It should be noted that the appearance of emission feature of crystalline silicate could be sensitive to not only the mass fraction of crystalline silicate but also the spatial distribution of the temperatures of the carrier of crystalline silicate as well as the amorphous silicate grain.

We conclude that the crystallization of silicate is a natural consequence of the formation process of dust grains prevailing in circumstellar envelopes of oxygen-rich AGB stars. The crystalline silicate is a diagnostics of high mass loss rate at the late stage of AGB stellar evolution. The problem of Fe content in the crystalline silicate suggested by the observations (Tielens et al. 1998) has been left for the future; we may speculate that the thermal processing at the temperature above $1000 \mathrm{~K}$ could make crystalline silicate $\mathrm{Mg}$-rich. The crystalline silicate could be less active for reaction with Fe than small cluster of homogeneous silicate, which causes homogeneous silicate grains to be Fe-rich.

Acknowledgments. We are grateful to Dr. C. Koike for providing us the unpublished data of optical constants of crystalline olivine.

\section{References}

Avrami M., 1939, J. Chem. Phys. 7, 1103

Cami J., de Jong T., Justtanont K., et al., 1998, Ap\&SS 255, 339

Hallenbeck S. L., Nuth III J.A., Daukantas P., 1998, Icarus 131, 198

Koike C., 1998, private communication

Kouchi A., Yamamoto T., Kozasa T., et al., 1994, A\&A 290, 1009

Kozasa T., Sogawa H., 1997, Ap\&SS 251, 165

Kozasa T., Sogawa H., 1998, Ap\&SS 255, 437

Seki J., Hasegawa H., 1981, Prog. Theor. Phys. 66, 903

Tielens A.G.G.M., Waters L.B.F.M., et al., 1998, Ap\&SS 255, 415

Waters L.B.F.M., Molster F.J., de Jong T., et al., 1996, A\&A 315, L361 